\title{
Das Koordinatensystem der Methodik
}

\author{
Tonio Walter *
}

Immer wieder stehen Juristen vor den Fragen, was bei einer Auslegung zulässig sei, was die Auslegung von Analogien und teleologischen Reduktionen unterscheide, wie weit man die Vorstellungen des Normgebers zu respektieren habe und woraus sich der Sinn einer Norm erschliesse. Der folgende Beitrag spürt diesen methodischen Grundsatzfragen nach. Geschrieben wurde er ursprünglich für die Teilnehmer eines deutsch-schweizerischen Doktorandenseminars zur Methodik. Er verfolgt daher vorrangig ein didaktisches Ziel und hat nicht den Ehrgeiz, jenes Mass an Belegen aus Schriftum und Rechtsprechung beizubringen, das in einem rein wissenschaftlichen Aufsatz wünschenswert wäre.

I. Dimensionen juristischer Methodik.......................................................... 139

II. Die Werkzeuge der klassischen Dogmatik und ihre Anwendung ....................... 139

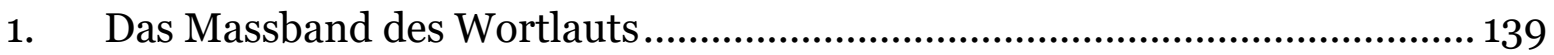

a) Der Begriff der Wortlautgrenze................................................................. 140

b) Die methodische Relevanz der Wortlautgrenzen ......................................140

c) Die Unschärfe und das zeitbedingte Wandern der Wortlautgrenze .............. 142

d) Wie ist die Wortlautgrenze zu ermitteln? ................................................ 143

2. Die Werkzeuge der Auslegung ............................................................ 145

a) Aspekte sind keine Gründe - zum Unterschied von topoi und argumenta ... 145

b) Der Wille des aktuellen Gesetzgebers als Auslegungsziel ............................ 146

c) Schranken für die Verwirklichung des gesetzgeberischen Willens ................ 149

d) Wie lässt sich der Wille des Gesetzgebers ermitteln?.................................151

III. Fazit: Wortlautgrenzen, Empirie und der Wille des Normgebers ..................... 153

Zitiervorschlag: Tonio Walter, Das Koordinatensystem der Methodik, in: sui-generis 2019, S. 138

URL: $\quad$ sui-generis.ch/99

DOI: $\quad$ https://doi.org/10.21257/sg.99

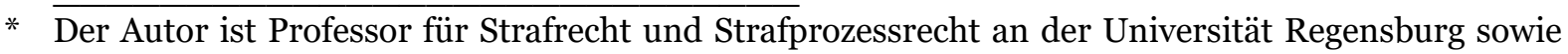
Richter am Bayerischen Obersten Landesgericht (tonio.walter@jura.uni-regensburg.de). Dem Schweizer Mitveranstalter Marc Thommen (Zürich), meinen Dank für seine Kommentare und Anregungen, die ich für diese Endfassung berücksichtigt habe. 


\section{Dimensionen juristischer Methodik}

1 Ein Koordinatensystem hat mindestens zwei Dimensionen, üblicherweise als $\mathrm{x}$ und $y$-Achse bezeichnet. In der juristischen Methodik lassen sich wenigstens fünf solcher Dimensionen unterscheiden. Da ist zum ersten die der Akteure: Wer ist juristisch tätig? Der Gesetzgeber? Ein Richter? Die Verwaltung? Mit einer Auswechslung des Akteurs muss zwar nicht zwangsläufig auch eine Änderung der Methoden verbunden sein, kann es aber. Etwa liegt auf der Hand, dass die Methodik eines Gesetzgebers etwas anderes ist als die Methodik des Rechtsanwenders. Das leitet über zur zweiten methodischen Dimension, das ist ihr Gegenstand, mit anderen Worten das Feld, auf dem die gesuchten Ergebnisse liegen. Hauptsächlich sind dies die Rechtsetzung, die Rechtsanwendung und das Rechtstatsächliche. Diese Gegenstände werden nicht unbedingt von der Person des methodischen Akteurs vorgegeben. Etwa ist ein Gesetzgeber zwar vorrangig rechtsetzend tätig, oft aber zugleich rechtsanwendend; und zwar dann, wenn ihm höherrangiges Recht Vorgaben macht, namentlich das Verfassungsrecht. Drittens hat die Methodik eine geschichtliche Dimension: Die Methodengeschichte ist Teil der Rechtsgeschichte. ${ }^{1}$ Viertens hat auch die Methodik eine internationale Dimension: Ein Rechtsvergleich kann - und sollte - auch ein Methodenvergleich sein. ${ }^{2}$

2 Die fünfte und wichtigste Dimension der Methodik sind ihre Werkzeuge. Das sind jene Pläne, Anleitungen und Wege, die zum juristischen Ziel führen sollen. Grob lassen sich zwei Werkzeugkästen unterscheiden: einmal die klassische Dogmatik, in der es darum geht, Rechtssätze auf einen Sachverhalt anzuwenden, also zu überlegen, welche Rechtsfolgen der Sachverhalt gemäss jenen Rechtssätzen habe. Und zum zweiten alle anderen Methoden der Rechtswissenschaft, das dürften vor allem, wenn nicht ausschliesslich, empirische Methoden sein - von der rechtsgeschichtlichen Forschung über die ökonomische Analyse des Rechts bis hin zur Kriminologie. Im Folgenden geht es allein um die klassische Dogmatik, da sie der Werkzeugkasten ist, den die allermeisten Juristen zu benutzen haben.

\section{Die Werkzeuge der klassischen Dogmatik und ihre Anwendung}

\section{Das Massband des Wortlauts}

3 Der klassischen Dogmatik geht es darum, ob ein Sachverhalt dem Tatbestand eines bestimmten Rechtssatzes unterfalle. Um das zu entscheiden, hat man in einem ersten Schritt die Wortlautgrenze des Tatbestandes abzustecken.

\footnotetext{
Ein schweizerisch-deutsches rechtsvergleichendes Beispiel aus Schweizer Sicht liefern Puricel, Christian, und Schenkel, Silvan, Methodendiskussion im deutschen und schweizerischen Verfassungsrecht aus historischer Sicht, in: Juana Vasella und Anne-Sophie Morand (Hg.), Werte im Recht - Das Recht als Wert. Junge Rechtswissenschaft Luzern, Schulthess 2018, S. 3-22.
}

2 Hierzu als Beispiel erneut der Beitrag von Puricel/Schenkel (Fn. 1); ferner allgemein Hager, Günter, Rechtsmethoden in Europa, 2009, sowie Walter, Tonio, und Stagl, Jakob Fortunat, Auf dem Weg zu einer europäischen Methodenlehre? Juristische Methodik im Rechtsvergleich, JZ 2016, 892-895 (Tagungsbericht). 


\section{a) Der Begriff der Wortlautgrenze}

Unter der Wortlautgrenze versteht man zunächst einmal die Grenze zwischen Sachverhalten, die ein Wort noch bezeichnen kann, und solchen, bei denen dies nicht der Fall ist. Zum Beispiel kann das Wort «Mensch» in der Standardsprache nicht nur den geborenen und lebenden Menschen bezeichnen, sondern - im Randbereich des Begriffs auch den noch nicht geborenen, aber schon gezeugten, sowie den bereits gestorbenen Menschen. Zwar gibt es in diesem Randbereich genauere Bezeichnungen: Den noch nicht geborenen Menschen nennt man «Embryo», ab der neunten Schwangerschaftswoche «Fötus»; den bereits gestorbenen Menschen bezeichnen wir als «Toten» oder «Leichnam». Aber das Wort «Mensch» betrachtet die Sprachgemeinschaft in diesen Fällen gleichwohl als nicht ganz falsch. Das ist schon daran zu erkennen, dass sich ein Toter als verstorbener und ein Fötus als noch nicht geborener Mensch definieren lässt. Diese beiden Sachverhalte liegen folglich noch diesseits der Grenzen des Wortes «Mensch» - wenn auch nahe diesen Grenzen. Klar jenseits von ihnen liegt zum Beispiel der Sachverhalt, den man als «Wachsfigur» bezeichnet; auch wenn eine Wachsfigur im Wachsfigurenkabinett grosse äussere Ähnlichkeit mit einem Menschen hat. Da dieser Sachverhalt jenseits der Wortlautgrenze liegt, könnte man auch sagen, es handle sich bei ihm um einen Teil des begrifflichen Aussenbereichs.

5 Eine Wortlautgrenze gibt es in der Rechtswissenschaft aber nicht nur als Abgrenzung zum begrifflichen Aussenbereich, sondern auch als Grenze zwischen den Sachverhalten, die ein Wort noch er- fassen kann, und jenen, die es bei jeder Betrachtung erfasst, also erfassen muss. Der Rechtswissenschaftler und berühmte Zivilrechtslehrer Philipp Heck hat die Sachverhalte, die ein Wort bei jeder Betrachtung erfasst, dessen Begriffskern genannt, und die weiteren Sachverhalte bis hin zur äusseren Wortlautgrenze - als Begriffshof.3 Zum Beispiel liegen die Sachverhalte «Embryo» und «Toter» im Begriffshof des Wortes «Mensch». In dessen Begriffskern bewegen sich alle geborenen und lebenden Exemplare der Spezies Homo sapiens.

\section{b) Die methodische Relevanz der Wortlautgrenzen}

6 Methodisch haben die beiden Wortlautgrenzen erhebliche Bedeutung. Das gilt auch für Rechtsordnungen, in denen die Gesetze - wie in der Schweiz - in mehreren Amtssprachen veröffentlicht werden. 4

\section{aa) Auslegung im Begriffshof}

7 Im Begriffshof der Normen spielt sich das ab, was man Auslegung nennt. Dort geht es um Sachverhalte - wie oben skizziert -, für die sprachlich nicht vollkommen klar feststeht, ob der Wortlaut der Norm sie erfasse. Diese Frage versucht man im Wege der Auslegung zu klären.5

3 Heck, Philipp, Gesetzesauslegung und Interessenjurisprudenz, AcP 112 (1914) S. 1 (46 f., 173); ders. Begriffsbildung und Interessenjurisprudenz, 1932, S. 52, 60. Zu diesem «Drei-BereicheModell» aus schweizerischer Sicht Kramer, Ernst A., Juristische Methodenlehre, 5. Auflage 2016, S. $62 \mathrm{ff}$.

4 Seelmann, Kurt, und Geth, Christopher, Strafrecht Allgemeiner Teil, 6. Auflage 2016, Rn. 85. In der Europäischen Union hat man es mit 24 Amtssprachen zu tun.

5 Zur Auslegung aus schweizerischer Sicht Kramer (Fn. 3) S. 57-189; aus deutscher Sicht Wank, Rolf, Die Auslegung von Gesetzen, 6. Auflage 
Ein strafrechtliches Beispiel ist die Auslegungsfrage, ob eine Langlaufloipe noch eine «Sache» sei im Sinne des Straftatbestandes der Sachbeschädigung. ${ }^{6}$

\section{bb) Teleologische Reduktion im Begriffskern}

8 Im Begriffskern stellen sich keine Auslegungsfragen, weil der Wortlaut den fraglichen Sachverhalt klar erfasst. Dann ist methodisch indes noch eine sogenannte teleologische Reduktion möglich. Bei ihr tut man so, als erfasste der Wortlaut den fraglichen Sachverhalt nicht («Reduktion» des Wortlauts), und stützt sich dafür auf den Sinn und Zweck der Norm, mit einem Fremdwort: auf ihr Telos. ${ }^{7}$ Ein strafrechtliches Beispiel ist sowohl in der Schweiz als auch in Deutschland der Grundtatbestand der Tötungsdelikte, der in der Schweiz «vorsätzliche Tötung» heisst (Artikel 111 des schweizerischen Strafgesetzbuches) und in Deutschland «Totschlag» (§ 212 des deutschen Strafgesetzbuches). Denn in beiden Ländern heisst es dort nur: Wer «einen Menschen tötet», wird bestraft; und das erfasst sprachlich auch die Selbsttötung. Sie ist aber in beiden Ländern unstreitig tatbestandslos. Der methodische Weg zu diesem Ergebnis ist die teleologische Reduktion - auch wenn das kaum einmal ausgesprochen wird. Richtig ist diese Reduktion, weil sie dem klaren Willen des historischen wie auch des aktuellen Gesetzgebers entspricht.

2015; rechtsgeschichtlich und rechtsvergleichend Hager (Fn. 2) S. 13-85.

6 Nein, meint BayObLG NJW 1980, 132 (f.); ja, meint Michael J. Schmid in seiner Anmerkung dazu in JR 1980, 430 (f.); ebenso die Vorinstanz.

Näher Kramer (Fn. 3) S. 233-247.

\section{cc) Analogie im begrifflichen Aussenbereich}

9 Im begrifflichen Aussenbereich ist ebenfalls keine Auslegung möglich, weil klar ist, dass der Wortlaut der Norm den Sachverhalt nicht erfasst. Dann stellt sich aber noch die Frage, ob man die Norm im Wege einer Analogie, das heisst entsprechend anwenden solle. Hierbei tut man so, als erfasste der Wortlaut den Sachverhalt noch, und dafür beruft man sich wieder auf den Normzweck - mit der zusätzlichen Voraussetzung, dass eine «planwidrige Regelungslücke» vorliegt, das heisst dass der Gesetzgeber die Norm versehentlich nicht auf den fraglichen Sachverhalt erstreckt hat und dies getan hätte, wenn man ihn auf jenen Sachverhalt hingewiesen haben würde. ${ }^{8}$ Bei Licht betrachtet ist dies allerdings im Vergleich zur teleologischen Reduktion kein zusätzliches Erfordernis, da auch die teleologische Reduktion nur zulässig ist, wenn sie zu den Plänen des Gesetzgebers passt, der Gesetzgeber also den fraglichen Fall selbst aus dem Wortlaut der Norm herausgenommen haben würde, wenn ihn jemand auf das Problem hingewiesen hätte. Daher handelt es sich bei teleologischer Reduktion und Analogie um vollständig komplementäre Methoden. Beispiel einer Analogie ist im deutschen Öffentlichen Recht, dass den Bürgern die Rechtsweggarantie des Artikels 19 Absatz 4 des Grundgesetzes nicht nur dann zugestanden wird, wenn sie in ihren Rechten tatsächlich verletzt worden sind - wie es der Wortlaut der Norm verlangt -, sondern natürlich und gerade

8 Näher Kramer (Fn. 3) S. 211-233; Müller, Friedrich, und Christensen, Ralph, Juristische Methodik, Band I: Grundlegung für die Arbeitsmethoden der Rechtspraxis, 11. Auflage 2013, Rn. 371 (S. 393-398). 
auch dann, wenn dies nur als möglich erscheint. 9 Ein Beispiel aus dem schweizerischen Recht betrifft die Pächter von Grundstücken, die man analog Artikel 679 ZGB wie Eigentümer haften lässt, wenn sie jemanden durch ein Überschreiten ihres Pachtrechts schädigen oder zu schädigen drohen. ${ }^{10}$

Im Strafrecht hat die Abgrenzung von Auslegung und Analogie sowie teleologischer Reduktion eine grössere Bedeutung als im Zivilrecht, da im schweizerischen wie auch im deutschen Strafrecht Analogien zulasten der Rechtsunterworfenen verboten sind. Dass dieses Verbot auch für teleologische Reduktionen gilt, ist in Deutschland mittlerweile anerkannt. ${ }^{11}$ Für die Schweiz habe ich eine solche Aussage noch nicht gefunden; sie läge aber ebenso nahe wie für das deutsche Strafrecht. Im sonstigen Öffentlichen Recht gilt in keinem der beiden Länder ein generelles Analogieverbot zulasten der Bürger. Für bestimmte Eingriffsbefugnisse ist es aber aus den gleichen Gründen wie im Strafrecht zu erwägen. ${ }^{12}$

\section{c) Die Unschärfe und das zeitbe- dingte Wandern der Wortlaut- grenze}

11 Sowohl die Grenze zwischen dem Begriffskern und dem Begriffshof als auch jene zwischen dem Begriffshof und dem

Siehe nur Jarass, Hans D., und Pieroth, Bodo, Grundgesetz für die Bundesrepublik Deutschland. Kommentar, 13. Auflage 2014, Art. 19 Rn. 41 (dort nicht als Analogie bezeichnet, in der Sache handelt es sich aber um einen Analogieschluss).

10 BGE 104 II 15.

11 Seit BGHSt. 42, 235 (241 f.).

12 Näher Bach, Markus, Das Analogieverbot im Verwaltungsrecht, 2011; Beaucamp, Guy, Zum Analogieverbot im öffentlichen Recht, AöR 134 (2009) S. 83-105.
Begriffsaussenbereich sind keine scharfen Grenzen in dem Sinne, dass sich für jeden Sachverhalt zweifelsfrei bestimmen liesse, ob er diesseits oder jenseits der Wortlautgrenzen liege. Vielmehr gibt es stets einen begrifflichen Graubereich von Sachverhalten, über die sich streiten lässt. Es verhält sich insoweit wie mit der Grenze zwischen Tag und Nacht, die sich zumindest für jemanden ohne Messgeräte nicht sekundengenau bestimmen lässt, weil die Dämmerung für eine längere Übergangszeit sorgt. Gleichwohl ist irgendwann der Zeitpunkt erreicht, in dem der Beobachter keine Zweifel mehr hat, dass es nunmehr Tag sei, weil die Sonne hell scheint, beziehungsweise Nacht, weil es stockfinster ist. Und genauso gibt es stets Sachverhalte, die nach jeder Betrachtung klar ausserhalb dessen liegen, was ein bestimmtes Wort bezeichnen kann, beziehungsweise klar innerhalb seines Bedeutungsfeldes.

So, wie sich die Grenze zwischen Tag und Nacht über das Jahr hin verschiebt vom längsten bis hin zum kürzesten Tag und zurück -, so kann sich auch eine Wortlautgrenze verschieben, weil sich die Sprache wandelt: Wörter haben nicht durch alle Zeiten hindurch Bestand, und sie haben auch nicht durch alle Zeiten hindurch die gleiche Bedeutung. Ein Beispiel ist der Begriff der «Wartung», der früher auch die Pflege von Menschen umfasste, ${ }^{13}$ während dieses Wort heute nur noch die Pflege von Maschinen und sonstigen technischen Einrichtungen bezeichnen kann.

13 Siehe etwa im Preussischen Allgemeinen Landrecht von 1794 die $\S \S 63,66$ («Pflege und Wartung» der Eltern durch die Kinder beziehungsweise der Kinder durch die Mutter). 


\section{d) Wie ist die Wortlautgrenze zu ermitteln?}

Angesichts der methodischen Bedeutung der Wortlautgrenze lautet die entscheidende Frage: Wie ist die Wortlautgrenze zu ermitteln? Gilt ein Gesetz in mehreren Amtssprachen, stellt sich diese Frage für jede von ihnen gleichermassen. Sie führt stets zu zwei Unterfragen, erstens: Welche (wie Sprachwissenschaftler sagen) Sprachvarietät ist zu untersuchen - die Fachsprache? Die Standardsprache? Die Umgangssprache? Zweitens: Welches ist das richtige Instrument, um die Wortlautgrenze zu ermitteln - ein Wörterbuch? Eine Umfrage? Die eigene Sprachkompetenz?

\section{aa) Welche Sprachvarietät ist massgeblich?}

14 Diese Frage dürfte in Abhängigkeit davon $\mathrm{zu}$ beantworten sein, an wen sich eine Norm wendet, wer also ihr Adressat ist. ${ }^{14}$ Handelt es sich um eine verwaltungsinterne Richtlinie, wird man ohne weiteres auf die juristische Fachsprache abstellen dürfen. Geht es um das Berufsrecht der Ärzte, können medizinische Fachbegriffe ohne Bedenken herangezogen werden. Steht die Norm hingegen als Allgemeindelikt im Strafgesetzbuch - und nicht als Sonderdelikt, das nur bestimmte Personen, etwa Amtsträger verwirklichen können -, so muss sich ihr Wortlaut an der Standardsprache messen lassen, und zwar an jener der Gegenwart. 15

14 Für Deutschland BVerfGE 126, 170 (197); 92, 1 (12); Lorenz, Jörn, Pietzcker, Manja, und Pietzcker, Frank, Empirische Sprachgebrauchsanalyse Entlarvt ein neues Beweismittel Verletzungen des Analogieverbots (Art. 103 II GG)?, NStZ 2005, 429-434 (430).

15 Für Deutschland BVerfGE 130, 1 (43); 71, 108 (115); Eser/Hecker in Schönke/Schröder, Strafgesetzbuch, Kommentar, bearb. v. Albin Eser u. a.,

\section{bb) Welches Instrument ist zu verwenden?}

15 Schwieriger ist die zweite Frage zu beantworten: Mit welchem Instrument ist die Wortlautgrenze zu ermitteln? Hierauf gibt Hans Kudlich eine verwirrende Antwort, denn er schreibt: durch Auslegung. ${ }^{16} \mathrm{Er}$ begründet das unter anderem damit, dass Straftatbestände zum Teil das Handlungsobjekt des Täters (Tatobjekt) nur in der Mehrzahl (Plural) nennen, obwohl unstreitig auch die Tat mit nur einem Handlungsobjekt erfasst werden soll und muss; zum Beispiel in $\S \S 152 a$ und 152b des deutschen Strafgesetzbuches, in denen unter anderem eine Fälschung von Zahlungskarten und Schecks für strafbar erklärt wird - obwohl nach ganz herrschender Ansicht auch die Fälschung nur einer Zahlungskarte und nur eines Schecks tatbestandsmässig ist. ${ }^{17}$ Daraus folgert Kudlich, dass nicht allein sprachliche Regeln, hier die Grammatik, die Grenzen des Wortlautes bestimmten. Und daraus wiederum schliesst er, dass sich auch diese Grenzen einer Auslegung verdankten.

16 Das ist allerdings eine Folgerung, die im begrifflichen Koordinatensystem der Methodik keinen Platz findet. Denn sie verletzt das Gebot, dass eine Fachsprache so widerspruchsarm wie möglich bleiben muss. Der Widerspruch, um den es hier geht, liegt darin, dass die Wortlautgrenze

29. Auflage 2014, § 1 Rn. 54; Roxin, Claus, Strafrecht Allgemeiner Teil, Band I, 4. Auflage 2006, $\S 5$ Rn. 26 ff., 28; wohl auch BGHSt. 52, 89 Rn. 8.

16 Kudlich, Hans, «Regeln der Grammatik», grammatische Auslegung und Wortlautgrenze, in: Hans-Ullrich Paeffgen u. a. (Hg.), Strafrechtswissenschaft als Analyse und Konstruktion. Festschrift für Ingeborg Puppe zum 70. Geburtstag, Duncker \& Humblot 2011, S. 123-136 (129 f.).

17 Grundlegend BGHSt. 46, 147 (150 f.). 
das Feld der Auslegung beschränkt, siehe oben $\mathrm{b}$, und dass sie daher nicht ihrerseits durch Auslegung zu ermitteln sein kann: Dürfte die Auslegung ihre Grenzen selbst festlegen, hätte sie keine. Und das ist nicht nur ein begrifflicher Widerspruch, sondern dahinter steht ein handfester Interessengegensatz, den es abzubilden gilt: Bei der Auslegung geht es darum - wie noch zu zeigen sein wird -, den Willen des Gesetzgebers zu verwirklichen; bei der Wortlautgrenze darum, die Bürger vor der schrankenlosen und unvorhersehbaren Verwirklichung dieses Willens zu schützen. Noch pointierter: Die Auslegung findet zugunsten des Gesetzes statt, die Definition der Wortlautgrenze hingegen zugunsten derer, die dem Gesetz unterworfen sind. Das sind zwei konträre Rationes, und die lassen sich nicht mit einer einheitlichen Methode verwirklichen.

Das muss man aber auch nicht. Das Problem, vor das sich Kudlich gestellt sieht, schwindet, wenn man bedenkt, dass die Grammatik Wortbedeutungen zwar in aller Regel beeinflusst, aber nicht immer. So ist ein «studierter Jurist» niemand, der studiert worden ist, sondern jemand, der selbst studiert hat. Auch im übrigen, will sagen abgesehen von der Frage einer Wortbedeutung, machen wir ständig «Grammatikfehler»: Wir sagen «tausendundeine Nacht», obwohl es doch eigentlich «Nächte» heissen müsste, wir genehmigen uns «ein bis zwei Cocktails», obwohl die Regeln der Grammatik «einen Cocktail bis zwei Cocktails» verlangten - und anderes mehr. Auch für die grammatischen Regeln gilt folglich, dass sie Ausnahmen haben. Und eine dieser Ausnahmen sind die Tatbestände von Normen. Dort bringt der Plural das Abstrakt-Generelle der Norm zum Ausdruck, was schon einfachste Beispiele verdeutlichen: «Fahrräder abstellen verboten!» heisst es, wenn dies eine für alle geltende Norm sein soll. Genau so werden diese Worte auch verstanden, und es käme niemand auf die Idee, dass lediglich das Abstellen von mindestens zwei Fahrrädern verboten wäre. Die grammatischen Grundregeln sind nun einmal nicht mehr als dies: Grundregeln, und sie kennen Ausnahmen. Wenn es aber nicht eine Auslegung ist, mit der sich die Grenzen des Wortlauts ermitteln lassen, stehen wir weiterhin vor der Frage, welches Instrument hierfür geeignet sei?

\section{cc) Wörterbücher versus empirische Sprachge- brauchsermittlung}

18 Bislang ziehen deutsche Gerichte die grossen Wörterbücher heran, wenn sie sich über die Grenzen und Einzelheiten einer Wortbedeutung informieren wollen: den Duden, den Wahrig, das Grimmsche Wörterbuch sowie grosse Enzyklopedien. ${ }^{18}$ Früher taten dies auch die US-amerikanischen Gerichte.19 Diese Methode ist aber fragwürdig. Denn Wörterbücher stammen herkömmlich aus der Feder einzelner Autoren, die auch nicht offenlegen, wie sie zu ihren Definitionen und Beispielen kommen. Und sie wollen lediglich positive Beispiele für den Wortgebrauch nennen, haben aber nicht den

18 Vgl. BVerfGE 73, 206 (243) (Duden); BGHSt. 52, 89 Rn. 8 (Duden und Grimmsches Wörterbuch); BGH (Z) NJW 1967, 343 (346) (Grimmsches Wörterbuch, Etymologisches Wörterbuch von Kluge/Götze, Trübners Deutsches Wörterbuch, Der grosse Brockhaus, Bülows Wörterbuch der Wirtschaft sowie Gablers Wirtschaftslexikon).

19 Vgl. Muscarello vs. United States 524 U. S. 125 (1998). 
Anspruch, damit die Wortbedeutung abschliessend festzulegen, das heisst auch negativ zu definieren (nach dem Motto «alles, was hier nicht steht, ist falsch»). Aufgrund dieser Schwächen der Wörterbücher ist die US-amerikanische Rechtsprechung dazu übergegangen, ein anderes Hilfsmittel zu verwenden: die Korpuslinguistik. ${ }^{20}$ Sie zieht eine möglichst grosse und repräsentativ zusammengestellte Textmenge heran, den sogenannten Textkorpus, und ermittelt dann mit der Suchfunktion eines Rechners, wie dort die Wörter verwendet werden. ${ }^{21}$ Das ist die eine Möglichkeit der - so der Fachbegriff - empirischen Sprachgebrauchsanalyse. Die zweite Möglichkeit sind umfrageartige Erhebungen zum Sprachverständnis (demoskopische Methode).

19 Natürlich fragt sich, welche Anforderungen an demoskopische Erhebungen zu stellen sind und welche an die Zusammenstellung und Grösse untersuchter Textkorpora. Ferner wird man Mindesthäufigkeiten festlegen müssen, da es für einen rechtlich relevanten Sprachgebrauch nicht genügen kann, dass ihn irgendwer irgendwann einmal praktiziert hat. Doch diese Schwierigkeiten lassen

20 Im Anschluss an Mouritsen, Stephen C., The Dictionary Is Not a Fortress: Definitional Fallacies and a Corpus-Based Approach to Plain Meaning, BYU Law Review, Vol. 2010 Nr. 5, S. 1915-1979, obergerichtlich erstmals in In re Baby E.Z., Utah 266 P. 3d 702; später obergerichtlich in People $v$. Harris, Michigan 885 N. W. 2 d 832.

${ }^{21}$ Vgl. Hamann, Hanjo, Der «Sprachgebrauch» im Waffenarsenal der Jurisprudenz, in: Friedemann Vogel (Hg.), Zugänge zur Rechtssemantik. Interdisziplinäre Ansätze im Zeitalter der Mediatisierung, 2015, S. 184-204; Vogel, Friedemann, Pötters, Stephan, und Christensen, Ralph, Richterrecht der Arbeit - empirisch untersucht. Möglichkeiten und Grenzen computergestützter Textanalyse am Beispiel des Arbeitnehmerbegriffs, 2015, S. 72-79. sich bewältigen, und insgesamt erscheint eine empirische Ermittlung des Sprachgebrauchs heute als der plausibelste Weg, um die Grenzen eines Wortlauts abzuschreiten. Hat man das getan, rücken die Instrumente der Auslegung in den Blick.

\section{Die Werkzeuge der Auslegung}

\section{a) Aspekte sind keine Gründe - zum Unterschied von topoi und argumenta}

2o Der gängigen Methodik fallen zum Thema Auslegung zunächst vier klassische Auslegungsarten ein: die grammatische, die historische, die systematische und die teleologische Auslegung. Man führt diesen Kanon der Auslegungsarten auf Savigny zurück (mit zweifelhafter Berechtigung). ${ }^{22}$ Hinzu kommen Variationen, wenn höherrangiges Recht Berücksichtigung verlangt: die verfassungskonforme Auslegung, die europarechtsfreundliche oder -konforme, in Deutschland insbesondere die richtlinienkonforme Auslegung sowie die völkerrechtsfreundliche oder -konforme Auslegung. Für einen begrifflichen Mangel halte ich es, dass man diese Auslegungsarten zugleich als Begründungen betrachtet und dann zum Beispiel sagt, die historische Auslegung führe zu diesem oder jenem Ergebnis. Denn das vermischt unausgesprochen zwei Dinge, die man zu trennen hat: einmal die unterschiedlichen Gesichtspunkte (Aspekte), die es zu berück-

${ }_{22}$ Siehe Savigny, Friedrich Carl von, System des heutigen römischen Rechts, Bd. 1, 1840, S. 206 ff., und dazu Hohmann, Hanns, in: Gerd Ueding (Hg.), Historisches Wörterbuch der Rhetorik, Bd. 4, Niemeyer 1998, Stichwort «Juristische Rhetorik» unter B. IV.; meine Kleine Rhetorikschule für Juristen, 2. Auflage, C. H. Beck 2017, S. $215 \mathrm{ff}$. 
sichtigen gilt, etwa die Gesetzessystematik; und zum zweiten die Begründungen (Argumente), die sich mit Blick auf einen solchen Gesichtspunkt gewinnen lassen, etwa ein Umkehrschluss, wenn der Blick auf die Gesetzessystematik ergibt, dass ein bestimmter Fall in vier von fünf gleichartigen Vorschriften ausdrücklich erwähnt wird, in der fünften aber nicht.

Beides $\mathrm{zu}$ trennen ist deshalb sinnvoll, weil jedenfalls drei der vier klassischen Auslegungsarten nur Topoi sind und keine Argumente: Die historische Auslegung ist nur ein Fingerzeig auf das, was man sich ansehen soll, und gleiches gilt für die grammatische und für die systematische Auslegung. Einzig die teleologische Auslegung scheint bereits ein Argument zu sein. Denn wenn man weiss, was der Sinn und Zweck eines Gesetzes ist, dann hat man oft auch schon eine Antwort auf die Frage, ob der in Rede stehende Fall vom Normtatbestand erfasst werden soll oder nicht. Doch das ist nicht immer so. Wenn ich zum Beispiel weiss, dass es der Normzweck einer Geringwertigkeitsklausel beim Diebstahlstatbestand ist, Strafverfahren wegen Bagatelltaten zu vereinfachen oder $\mathrm{zu}$ verhindern - dann weiss ich noch lange nicht, ab welcher Wertgrenze eine solche Geringwertigkeit anzunehmen ist, wenn sie mir das Gesetz nicht klar beziffert; und das tut es weder in Deutschland noch in der Schweiz, siehe $\S 248 \mathrm{a}$ des deutschen und Artikel $172^{\text {ter }}$ des Schweizer StGBs.

\section{b) Der Wille des aktuellen Gesetz- gebers als Auslegungsziel}

\section{aa) Die alte Frage nach dem Rang der Auslegungsarten}

22 Auch wenn man sich klargemacht hat, dass eine Auslegungsart noch keine Begründung ist, bleibt die entscheidende Frage offen, welches Ziel man eigentlich bei der Auslegung verfolge: das gerechteste Ergebnis zu finden? Oder das zeitgemässeste? Oder umkehrt das vom historischen Gesetzgeber gewollte? Oder das Ergebnis, das man selbst für das politisch erstrebenswerteste hält? Diese Frage ähnelt stark einer anderen, die man sich seit jeher in der Methodenlehre stellt, und zwar der Frage nach dem Rangverhältnis der Auslegungsarten: Gibt es eines? Und, falls ja: Welcher Auslegungsart gebührt der höchste Rang? Praktisch relevant wird diese Traditionsfrage der Methodik, wenn die einzelnen Auslegungsmodi - in Verbindung mit Argumentationsmustern und Sachgründen - in unterschiedliche Richtungen deuten. Die häufigste und plausibelste Antwort lautet, dass der teleologischen Auslegung die Krone gebühre:23 Der Sinn und Zweck des Gesetzes sei das Entscheidende. Dann schliesst sich allerdings sofort die weitere methodische Traditionsfrage an, wie dieser Sinn und Zweck zu bestimmen sei: als jener, den der historische Gesetzgeber wollte? Oder als objektiver Gesetzeszweck?

23 So für die Schweiz Kramer (Fn. 3) S. 187 ff.; für Deutschland Jescheck, Hans-Heinrich, und Weigend, Thomas, Lehrbuch des Strafrechts. Allgemeiner Teil, 5. Auflage, Duncker \& Humblot 1996, § 17 IV 1 b (S. 156). 


\section{bb) Der Wille des aktuellen Gesetz- gebers als massgebliches Auslegungsziel}

23 Für alle diese Fragen gibt es meines Erachtens nur eine Lösung: Als Ziel der Auslegung massgeblich ist allein der Wille des aktuellen Gesetzgebers; sein ausdrücklich erklärter Wille - oder der zu mutmassende, wenn es keine unmittelbar einschlägigen Äusserungen dieses Willens gibt. Demgegenüber dürfte die herrschende Auffassung dahin gehen in der Schweiz und in Deutschland -, einen objektiven Gesetzeszweck für massgeblich zu halten. Das ist aber lediglich eine Tarnung dafür, den eigenen Wertungen zum Durchbruch zu verhelfen. Um dies zu erkennen, hat man sich zunächst vor Augen zu halten, dass es keinen objektiven Sinn gibt, weder von Gesetzen noch von sonst etwas. Sinnstiftung ist stets das Privileg geistbegabter Wesen. Ohne ihre Sinnsetzung - und zwar durch einen rein geistigen Akt - gibt es keinen Sinn, sondern lediglich eine objektive Funktion und Wirkung.

24 Ein alltägliches Beispiel mag das verdeutlichen: Wer sich vor den Blicken seines Nachbarn schützen will, kann zum nachbarlichen Grundstück hin einen Zaun errichten. Wenn diese Arbeit fachgerecht ausgeführt wird, hat der Zaun hernach objektiv die Wirkung, Sichtschutz zu gewährleisten. Und weil das der Grund ist, weswegen er errichtet wurde, hat er auch diesen Sinn. Nun mag sein Erbauer feststellen, dass der Zaun ausserdem im Sommer Schatten spendet. Dies ist zunächst einmal lediglich eine weitere Wirkung des Zaunes. Ob es auch sein weiterer Zweck wird, entscheidet erneut sein Erbauer: Ist er sonnenempfindlich, wird er im Sommer den Schatten begrüssen und den Zweck des Zaunes fürderhin auch darin sehen, diesen Schatten zu spenden. Besonders deutlich wird dies, wenn der frühere Zweck «Sichtschutz» wegfällt, zum Beispiel weil das Nachbarhaus von dem Erbauer und Eigentümer des Zaunes gekauft und zugunsten einer Erweiterung seines Gartens abgerissen wird - und wenn er den Zaun dann gleichwohl stehen lässt um des Schattens willen. Dann ist das Schattenspenden von diesem Zeitpunkt an sogar der einzige Zweck des Zaunes, und die Sichtblockade wird zu einem unerwünschten Nebeneffekt, sinkt also zu einer ausschliesslich objektiven Wirkung herab. Und wenn der Zaunerbauer auszieht und ihm als Hausbewohner ein Sonnenanbeter folgt, der sich über den Schatten ärgert, verliert der Zaun sofort den Zweck des Schattenspendens - bekommt aber womöglich wieder den des Sichtschutzes, falls das andere Grundstück erneut von Dritten gekauft und bebaut wird. Der Zaun selbst steht während der gesamten Zeit äusserlich unverändert an der Grundstücksgrenze. Seinen Sinn und Zweck müssen ihm die Menschen geben.

25 Bei der Auslegung von Gesetzen gibt es aber nicht nur keinen objektiven Sinn, sondern auch keine objektive Wirkung oder Funktion. Denn es ist doch gerade die Frage, ob das Gesetz in dem fraglichen Fall wirke und funktioniere, das heisst den fraglichen Fall mit seinem Tatbestand erfasse. Und daher gibt es schlechterdings nichts, was man bei der Auslegung von Gesetzen als objektiven Zweck bestimmen könnte. Nichts - ausser den eigenen Vorstellungen von Vernunft und Gerechtigkeit. Und just die pflegen sich Bahn zu brechen, wenn je- 
mand bei der Auslegung auf den vermeintlich objektiven Sinn eines Gesetzes abstellt. Das muss zwar im Ergebnis nicht verwerflich sein, denn es gibt Fälle, in denen hat man als Auslegungshilfe trotz aller Bemühungen am Schluss nur diese eigenen Vorstellungen vom Vernünftigen und Gerechten. ${ }^{24}$ Aber das sind eben nur einige Fälle und längst nicht alle. Und das, was der Interpret dann tut, wird methodisch falsch etikettiert, wenn er sich auf objektive Sinnbestimmungen beruft.

Wenn es aber keinen objektiven Sinn gibt, sondern nur einen subjektiven, der von einer geistbegabten Person gestiftet werden muss, dann muss diese Person mit Blick auf unsere Gesetze deren Gesetzgeber sein. Dieses «muss» ist allerdings nicht im Sinne eines logischen Zwanges $\mathrm{zu}$ verstehen, sondern ist eine staats- und demokratietheoretische Forderung, die man erheben kann, aber nicht logisch zwingend zu erheben hat. Man kann auch der Ansicht sein, dass sich der Rechtsanwender von den Wünschen und Vorstellungen des Gesetzgebers zu emanzipieren habe, und zwar ganz gleich, ob es um den historischen Gesetzgeber geht oder um den aktuellen. Das ist die Position der sogenannten Freirechtsschule gewesen, mit der sich der deutsch-schweizerische Zivilrechtslehrer Hans Reichel in seinem Werk $G e$ setz und Richterspruch besonders gründlich auseinandergesetzt hat. ${ }^{25}$

24 Zutreffend schon Kramer (Fn. 3) S. 164 («Eigenwertung»).

25 Reichel, Hans, Gesetz und Richterspruch. Zur Orientierung über Rechtsquellen- und Rechtsanwendungslehre der Gegenwart, Orell Füssli 1915.
27 Wie man in dem Verhältnis von Gesetzgeber und Richter die Kompetenzakzente setzt, hat auch damit zu tun, welche Legitimation der Gesetzgeber hat und welche der Richter. Verfügen die Richter, wie mitunter in der Schweiz, über eine unmittelbare demokratische Legitimation, liegt es näher, ihnen gegenüber dem Gesetzgeber eine grössere Freiheit zuzugestehen als in Deutschland, wo die Richter nur sehr mittelbar und schwach demokratisch legitimiert sind. Umgekehrt könnte man in der Schweiz auf die Idee kommen, bei der Auslegung direktdemokratisch geschaffener Rechtssätze eine strengere Bindung an den Willen des (Volks-)Gesetzgebers anzunehmen als in anderen Fällen. Für zweifelhaft halte ich jedenfalls die in Deutschland und dort besonders unter Zivilrechtlern zu beobachtende Haltung, sich stärker als der Gesetzgeber berufen zu fühlen, das Richtige und Gerechte zu erkennen.

28

Erklärt man den Willen des Gesetzgebers zum höchsten Auslegungsziel, so bettet man den Gesetzgeber keineswegs weicher, als er ohnehin schon liegt. Im Gegenteil: Man nimmt ihn durch diese Auslegungsmaxime auch in die Pflicht. Schliesslich führt sie dazu, dass sehr genau zu ermitteln ist, was im Gesetzgebungsverfahren verhandelt wurde und wie sich die Gesetzesgenese erklären lässt; und nicht nur die des anzuwendenden Gesetzes, sondern zum Teil auch jene sachverwandter anderer Gesetze, dazu später erneut. Auch haben sich die Organe der Gesetzgebung darauf einzustellen, dass man alles, was sie im Verfahren der Gesetzgebung äussern, ernst und genau nehmen wird. Ferner müssen sie es sich gefallen lassen, dass man ihnen eine umfassende Kenntnis sowohl des gesamten 
übrigen Gesetzesbestandes unterstellt als auch der zu ihm ergangenen Rechtsprechung. Schliesslich noch sind dem Gesetzgeber im Zweifel sinnvolle, heisst widerspruchs- und redundanzfreie Gesetzgebungsakte zu unterstellen. Das bedeutet auch, dass die Auslegung einer Norm grundsätzlich keines ihrer Merkmale überflüssig machen darf (eine alte methodische Regel)26. Das wiederum verpflichtet den Gesetzgeber, mit besonderem Bedacht zu formulieren.

\section{c) Schranken für die Verwirkli- chung des gesetzgeberischen Willens}

\section{aa) Der Wortlaut}

29

Die Auslegung hat innerhalb der Grenzen des Wortlautes stattzufinden (oben 1). Sie sind nicht ihrerseits im Wege der Auslegung zu bestimmen, sondern nur durch eine grundsätzlich empirische Ermittlung des Wortgebrauchs.

\section{bb)Höherrangiges Recht}

3o Auch innerhalb der Grenzen des Wortlauts dürfen die Bemühungen, den Willen des Gesetzgebers zu verwirklichen, nie weiter reichen, als dieser Wille $\mathrm{zu}$ herrschen befugt ist. Grenzen zieht ihm der Wille eines höheren Gesetzgebers, folglich das höherrangige Recht. Mit Blick auf das nationale einfache Gesetzesrecht können solches höherrangige Recht das Verfassungsrecht und das Völkerrecht sein. Aber Achtung: Für den Anwender des einfachen Gesetzes ist das nur von Belang, soweit auch ihm erlaubt

$26 \overline{\text { Siehe bereits Ulpian, Dig. 2, 7, } 5 \text { § } 2 \text { a. E. und }}$ dazu Liebs, Detlef, Lateinische Rechtsregeln und Rechtssprichwörter, 7. Auflage, C. H. Beck 2007, unter V 9 (S. 242). ist, das höherrangige Recht unmittelbar und selbst gegen den Willen seines Gesetzgebers anzuwenden. In diesem Punkt gibt es deutliche Unterschiede zwischen der Schweiz und Deutschland. Während in der Schweiz jedes Gericht befugt ist, die Verfassung selbst gegen den Willen des einfachen Gesetzgebers in Stellung zu bringen, darf dies in Deutschland nur das Bundesverfassungsgericht. Das hat für die sogenannte verfassungskonforme Auslegung die gern übersehene Folge, dass diese Auslegung in Deutschland den Instanzgerichten verwehrt ist, sofern sie sich mit ihr gegen den Willen und die Ziele des Gesetzgebers stellen müssten ganz gleich, für wie verfassungswidrig ein Instanzgericht diesen Willen halten mag. ${ }^{27}$ Vielmehr hat es in einem solchen Fall die Sache gemäss Artikel 100 des Grundgesetzes dem Verfassungsgericht zur Entscheidung vorzulegen.

31 Ähnlich verhält es sich, wenn ein Gericht in der Schweiz oder in Deutschland meint, dass der Wille des nationalen Gesetzgebers der Europäischen Menschenrechtskonvention (EMRK) widerspreche, die beide Länder unterzeichnet und ratifiziert haben: Das Gericht darf den Willen des nationalen Gesetzgebers dann nicht für unbeachtlich halten, sondern hat das einfache Gesetzesrecht diesem Willen gemäss anzuwenden. Auch soweit die EMRK ihrerseits im Range eines einfachen Gesetzes gilt; und selbst dann, wenn es Rechtsprechung des Europäischen Gerichtshofes für Menschenrechte (EGMR) gibt, auf die sich das Gericht stützen könnte. Denn die Aufgabe, das nationale Recht konventionskonform auszugestalten, hat vorrangig der Gesetz-

27 BVerfGE 138, 296 (350); 86, 288 (320); 54, 277 (299). 
geber. Wo er dies zu tun versäumt, verhält er sich zwar völkerrechtswidrig und zieht seinem Land womöglich völkervertragliche Sanktionen zu. Aber das ist nicht das Problem der nationalen Gerichte. Und sie haben das nationale Recht zwar konventionskonform auszulegen. Aber eben nur auszulegen, und die Spielräume der Auslegung enden dort, wo es einen klar zum Ausdruck gebrachten gesetzgeberischen Willen gibt.

Dass der Wille des Gesetzgebers zurückzutreten hat, wenn ihm unmittelbar anwendbares höherrangiges Recht entgegensteht, gilt auch für die Analogie und die teleologische Reduktion. Sie sind also immer dann ausgeschlossen, wenn der gesetzgeberische Plan, auf den sich die Analogie zu stützen hätte, und das Telos, dem sich die Reduktion des Tatbestandes verdanken müsste, unmittelbar anwendbarem höherrangigem Recht widersprechen. Umgekehrt kann es sein, dass unmittelbar anwendbares höherrangiges Recht eine Analogie oder teleologische Reduktion erzwingt. Denn solches höherrangige Recht tritt nun einmal vollständig an die Stelle des gesetzgeberischen Willens. Es kann also auch eine verfassungskonforme Analogie und verfassungskonforme Reduktion geben; in Deutschland aber gegen den Willen des Gesetzgebers nur aus der Hand des Bundesverfassungsgerichts. Als - jetzt fiktives - Beispiel erneut den Straftatbestand der vorsätzlichen Tötung (Schweiz) beziehungsweise des Totschlags (Deutschland): Hätte der Gesetzgeber unmissverständlich kundgetan, dass er eine Strafbarkeit auch des Suizids wolle - was praktisch in den zahlreichen Fällen einer nur versuchten Selbsttötung von Belang wäre -, so dürfte in der Schweiz jedes
Gericht und in Deutschland immerhin das Bundesverfassungsgericht den Straftatbestand gleichwohl um den Fall des Suizids reduzieren, und zwar unter Berufung auf entgegenstehendes, unmittelbar anwendbares Verfassungsrecht.

\section{cc) Naturrecht?}

33 Unmittelbar anwendbares höherrangiges Recht ist auch das Naturrecht, von manchen «Vernunftrecht» genannt - wenn man denn meint, dass es dergleichen gebe. Ich meine das nicht; für tiefere Ausführungen zu diesem weiteren Ur-Thema der Methodik, ja der Rechtswissenschaft insgesamt ist hier kein Raum. ${ }^{28}$ Nur zu einem besonders berühmten und hier auch besonders einschlägigen Beispiel einige Worte, und zwar zur sogenannten Radbruchschen Formel. Sie ist gewissermassen negatives Naturrecht, und zwar eine Regel, mit der Gustav Radbruch $^{29}$ bestimmen wollte, wann Gesetze, obwohl juristisch in Geltung und praktisch angewandt, so ungerecht seien, dass man sie als nichtig anzusehen habe. Seine Antwort: wenn in ihnen «Gerechtigkeit nicht einmal erstrebt wird», wenn «die Gleichheit, die den Kern der Gerechtigkeit ausmacht, [...] bewusst verleugnet wird»30. Radbruchs Beispiel waren die Nürnberger Rassengesetze der Nationalsozialisten. Zwar hat seine Auffassung eine Schwäche: Der Kern der Gerechtig-

28 Näher etwa Dreier, Ralf, Recht - Moral - Ideologie, Suhrkamp 1981, S. 180 ff.; Weinberger, Ota, Norm und Institution. Eine Einführung in die Theorie des Rechts, Manzsche Verlags- und Universitätsbuchhandlung 1988, S. $116 \mathrm{ff}$.

$29 \mathrm{Zu}$ ihm Kaufmann, Arthur, Gustav Radbruch. Rechtsdenker, Philosoph, Sozialdemokrat, Piper 1987; auf knappstem Raum mein Editorial Von der Persönlichkeit des Juristen - zum 6o. Todestag Gustav Radbruchs, JA 2009, S. I.

3o Radbruch, Gustav, Gesetzliches Unrecht und übergesetzliches Recht, SJZ 1946, 105-108 (107). 
keit ist nicht irgendeine Gleichheit, sondern nur die gleiche Behandlung von wesentlich Gleichem - und was sich im wesentlichen gleicht, ist oft Ansichtssache. Doch ist diese Wertungsbedürftigkeit kein schlagender Einwand gegen Radbruchs Formulierung. Denn mittlerweile gibt es in Europa, oft sogar weltweit, eine breite Übereinstimmung über Umstände, die für die rechtliche Behandlung der Menschen ausser Betracht zu bleiben haben. Dies zeigt sich in den typischen Diskriminierungsverboten; etwa dürfen $\mathrm{Al}-$ ter, Geschlecht und ethnische Zugehörigkeit eines Menschen für seine Rechte grundsätzlich keine Rolle spielen.

34 Allerdings sind naturrechtliche Postulate der Radbruchschen Art gar nicht erforderlich, wenn man nach dem Ende einer Diktatur eine Handhabe sucht, um jene zu verfolgen, die mit staatlicher Lizenz Menschenrechte verletzt haben, und diejenigen zu rehabilitieren, die für die Wahrnehmung solcher Rechte verfolgt worden sind. Denn eine Alternative besteht darin, in solchen Fällen das Rückwirkungsverbot ausser Kraft zu setzen. Diesen Weg hat das Bundesverfassungsgericht in den Mauerschützenverfahren beschritten, also nach dem Ende des DDR-Regimes. ${ }^{11}$ Das erscheint vielen Strafrechtslehrern als Sakrileg. Es läuft aber auf das gleiche hinaus wie die Radbruchsche Formel.

Zwar lässt sich ein Unterschied anführen: Bei einer Aufhebung des Rückwirkungsverbots tritt die Strafbarkeit ex post ein, war also bei chronologischer Sicht zur Tatzeit nicht gegeben. Anders, wenn man die Radbruchsche Formel anwendet: Dann besteht die Strafbarkeit de iure von Anfang an und auch schon in den Augenblicken, in denen der Täter handelt, etwa ein Mauerschütze oder KZAufseher auf Flüchtende schiesst. Aber dieser Unterschied hat keine praktischen Folgen. Denn das Problem des Widerstandskämpfers ist nicht die Rechtslage, sondern die faktische Macht des Regimes. Was ihm Juristen und Rechtsphilosophen aus anderen Ländern zurufen, nützt ihm nichts, solange das Regime herrscht. Und danach kann es ihm einerlei sein, ob sich das Recht nur ex nunc, aber mit Rückwirkung ändert, oder ob es schon immer auf seiner Seite gestanden hat.

\section{d) Wie lässt sich der Wille des Gesetzgebers ermitteln?}

36 Der Wille eines Gesetzgebers ist insgesamt so zu ermitteln wie der des Geschäftsherrn bei der Geschäftsführung ohne Auftrag: Zuerst ist nach ausdrücklichen Willensäusserungen $\mathrm{zu}$ forschen, dann nach schlüssig zum Ausdruck gebrachten, und schliesslich, wenn sich überhaupt keine Botschaften oder Signale des Gesetzgebers finden lassen, hat man anzunehmen, dass er das gewollt haben würde, was objektiv vernünftig erscheint. Bei alldem kommt es nicht auf den historischen Gesetzgeber an, der vor Jahrzehnten oder Jahrhunderten tätig gewesen sein mag und womöglich Wertvorstellungen hatte, die uns heute fremd sind. Vielmehr geht es darum, den ausdrücklichen oder mutmasslichen Willen des gegenwärtigen Gesetzgebers zu ermitteln. Allerdings ist im Zweifel davon auszugehen, dass der Wille des historischen Gesetzgebers auch der des heutigen ist. Denn dass ein Wandel der Wertvorstellungen stattgefunden hat und sich

31 BVerfGE 95, 96 (133). 
auch noch auf eine Auslegungsfrage auswirkt, ist ein Ausnahmefall.

Ausdrückliche Bekundungen des gesetzgeberischen Willens finden sich vor allem in den Materialien des Gesetzes. In Betracht kommen aber auch andere Äusserungen der Legislativorgane. Ausserdem müssen die Materialien nicht unbedingt jene zu dem auszulegenden Gesetz sein; auch in den Begründungen anderer Gesetze lassen sich womöglich Hinweise auf Haltungen und Wertungen finden, die für die Auslegungsfrage eine Rolle spielen. Dass sich in den Materialien stets nur Äusserungen einzelner Akteure finden, vom Ministerialbeamten bis zum Abgeordneten, doch nie Äusserungen des Parlaments als Institution oder gar solche sämtlicher Abgeordneter, ist unschädlich. Erstens kommt es nie auf den Willen des Gesamtparlaments an, sondern stets nur auf den der parlamentarischen Mehrheit, die das Gesetz beschlossen hat. Zweitens sind dieser Mehrheit jene Äusserungen im Gesetzgebungsverfahren zuzurechnen, von denen sie sich offenbar hat überzeugen lassen. Zwar ist letzteres weitgehend eine Fiktion, aber eine legitime. Von ihr lebt die parlamentarische Demokratie. Auch solange es bei der Anwendung eines Gesetzes keine Auslegungsfragen gibt, wurzelt seine $\mathrm{Au}-$ torität darin, dass es im parlamentarischen Verfahren erörtert wurde und von der Parlamentsmehrheit auf der Grundlage dieser Erörterung beschlossen worden ist.

Sind keine ausdrücklichen Botschaften des Gesetzgebers zu finden, muss man versuchen, aus anderen Indizien auf seinen Willen zu schliessen. An dieser Stelle kommen die Topoi der klassischen Aus- legungsarten ins Spiel, denn solche Indizien können sich namentlich aus der Formulierung des Gesetzes, aus seiner Systematik und aus Vorgängernormen ableiten lassen. ${ }^{32}$ Die Gesetzessystematik kann auch insofern von Belang sein, als aus neueren Gesetzen an anderer Stelle auf Anliegen, Wünsche und Wertvorstellungen des aktuellen Gesetzgebers $\mathrm{zu}$ schliessen ist.

39 Natürlich bleiben die Bemühungen, den Willen des Gesetzgebers zu ermitteln, oft ohne Ergebnis: In den Materialien findet sich nichts oder gar Widersprüchliches, und auch sonst sind nirgends fallbezogene Indizien für einen hypothetischen Willen des Gesetzgebers zu entdecken. Doch das ist kein Grund, sich von vorneherein nicht um die Ermittlung dieses Willens $\mathrm{zu}$ bemühen. Wenn ein Weg schwierig wird oder an einem Abgrund endet, heisst dies nicht, dass der benutzte Kompass defekt wäre. Und eine Auslegungslehre kann nicht mehr sein als ein solcher Kompass. Sie ist kein Helikopter, der einen über alle Unwegsamkeiten hinweg stets sicher und auf der Luftlinie zum Ziel brächte.

40 Findet der Norminterpret auch keine konkludenten Indizien für den gesetzgeberischen Willen, darf und muss er das objektiv Vernünftige annehmen. Theoretisch ist dies allerdings noch immer nicht unbedingt das, was der Norminterpret für das Vernünftigste hält. Sondern er hat zu ermitteln, was nach allgemeiner, mindestens vorherrschender Auffassung vernünftig erscheint. Im wesentlichen ist das nichts anderes als die berühmte Regel, die Artikel 1 Absatz 2 ZGB an zweiter

32 Sehr ähnlich schon Kramer (Fn. 3) S. 162 ff. sowie BVerfGE 1, 299 (312). 
Stelle (zwar nicht für die Auslegung, aber) für das Füllen von Gesetzeslücken vorschreibt, also eine (hier) Auslegung modo legislatoris.

\section{Fazit: Wortlautgrenzen, Empirie und der Wille des Normgebers}

41 Zusammenfassen lässt sich das methodische Koordinatensystem, um das es mir geht, wie folgt: $\mathrm{Zu}$ allererst ist es erforderlich, die Wortlautgrenzen einer Norm zu bestimmen, und die erste Hauptbotschaft dieses Beitrages lautet, dass man dabei grundsätzlich empirisch vorzugehen hat: Das Ziel ist herauszufinden, wie die Normadressaten tatsächlich sprechen. Daraus folgen dann zwei Wortlautgrenzen, und zwar eine zum begrifflichen Aussenbereich, dessen Sachverhalte ein Wort nicht mehr erfassen kann, und eine zwischen dem Begriffshof und dem Begriffskern; wobei der Begriffskern aus jenen Sachverhalten besteht, die ein Wort ganz sicher erfasst. Im Begriffshof findet die Auslegung statt, im begrifflichen Aussenbereich ist nur eine Analogie möglich, und im Begriffskern - nur dort - kommt ausnahmsweise eine Umkehrung der Analogie in Betracht, das heisst eine teleologische Reduktion.

Die zweite Hauptbotschaft dieses Beitrages besagt, dass man bei der Auslegung nicht nur mit den Schlagworten der überkommenen Auslegungsarten operieren darf, weil diese Auslegungsarten lediglich Gesichtspunkte (Topoi) sind, an die gedacht werden muss, aber noch keine Argumente für ein bestimmtes Auslegungsergebnis. Vielmehr ergeben sich solche Argumente erst aus einer Verbindung von bestimmten Begründungsmustern, etwa dem Umkehrschluss, und den wahren Sachgründen, die im Beispiel des Umkehrschlusses dafür sprechen, diesen Schluss tatsächlich zu ziehen.

43 Die dritte Hauptbotschaft des Beitrages ist, dass die wahren Sachgründe solche sein sollten, die bemüht sind, den Willen des gegenwärtigen Normgebers zu verwirklichen - soweit dieser Wille zu herrschen befugt ist, das heisst soweit ihm kein höherrangiger Wille entgegensteht. Letzteres kommt allerdings nur in Betracht, wenn der Normanwender dafür zuständig ist, auch diesen höherrangigen Willen zur Geltung zu bringen, also höherrangiges Recht unmittelbar anzuwenden. $\mathrm{Zu}$ ermitteln ist der Wille des Normgebers wie jener des Geschäftsherrn bei der Geschäftsführung ohne Auftrag: An erster Stelle sind ausdrückliche Äusserungen des Gesetzgebers zu berücksichtigen, die vor allem in den Gesetzesmaterialien zu finden sein können. Als nächstes kommen konkludente Äusserungen des Gesetzgebers in Betracht - auch in Form einer Gesetzgebung und Gesetzesbegründung an anderer Stelle. Und wenn auch diese Suche erfolglos bleibt, darf und muss die Norm so ausgelegt werden, wie es der Vernunft entspricht, das heisst dem allgemein für vernünftig Gehaltenen.

44 Natürlich steckt bei alldem der Teufel im Detail. Aber bei einem Koordinatensystem geht es noch nicht um die Details, sondern um Dimensionen und Richtungen, in denen man zu denken hat. Dies dann anhand des Einzelfalles zu tun, ist eine zweite Aufgabe; und sicher die grössere. 